\title{
DC-based immunotherapy for hematological malignancies
}

\author{
Toshio Kitawaki
}

Received: 8 December 2013/Revised: 15 December 2013/Accepted: 16 December 2013/Published online: 31 December 2013

(C) The Japanese Society of Hematology 2013

\begin{abstract}
Great advances have been made in the treatment of hematological malignancies, but achieving a definitive cure remains an elusive goal for the majority of patients. Antigen-specific tumor immunotherapy has the potential to improve clinical outcome in patients with such diseases by eradicating chemotherapy-resistant tumor cell clones without damaging normal tissues. Dendritic cells (DCs) serve as an essential link between the innate and the adaptive immune systems, acting as key controllers of antigen-specific $\mathrm{T}$ cell responses. Molecular identification of tumor-specific antigens recognized by $\mathrm{T}$ lymphocytes and technical advances in ex vivo generation of human DCs has enabled us to develop DC-based tumor immunotherapies (also called "DC vaccines"). To date, a large number of clinical trials of DC vaccines have been conducted for a variety of tumors, including hematological malignancies. Overall, these trials have demonstrated that DC vaccines have excellent safety profiles, moderate immunological activity, and mild clinical efficacy. To establish a role for DC vaccines in the treatment of hematological malignancies, we need both to define patient populations that can obtain clinical benefit from DC vaccines and to develop combination therapies that augment clinical efficacy of DC vaccines. In this review, I will describe current status of DC-based immunotherapy for hematological malignancies, and discuss future perspectives in this field.
\end{abstract}

T. Kitawaki $(\bowtie)$

Department of Hematology and Oncology, Kyoto University Hospital, 54 Shogoin Kawara-cho, Sakyo-ku, Kyoto 606-8507, Japan

e-mail: kitawaki@kuhp.kyoto-u.ac.jp
Keywords Dendritic cells - Immunotherapy - DC vaccines $\cdot$ Clinical trials

\section{Introduction}

Although great advances have been made in the treatment of hematological malignancies, a definitive cure remains an elusive goal for the majority of patients. And even patients who obtained long-term disease-free survival often suffer from persistent adverse effects inflicted by cytotoxic chemotherapy. These problems arise from the inability of these therapies to eliminate chemotherapy-resistant tumor cell clones, especially tumor stem cells, and to discriminate tumor cells from normal tissues. Antigen-specific tumor immunotherapy, which has fine antigen specificity and different mechanisms of action from conventional therapeutic modalities, has the potential to improve clinical outcome of these patients by eradicating resistant clones without damaging normal tissues, and thus, is an attractive approach to overcome these problems. Among a variety of approaches to antigen-specific tumor immunotherapy, this review will focus on dendritic cell (DC)-based immunotherapy for tumors, especially for hematological malignancies.

\section{DC-based tumor immunotherapy}

Dendritic cell is an antigen-presenting cell (APC) that serves as an essential link between the innate and the adaptive immune systems [1]. DCs derive from hematopoietic stem cells, circulate in the peripheral blood, and travel to peripheral tissues, such as skin and mucosa, where they reside in an immature form (Fig. 1). When pathogens 


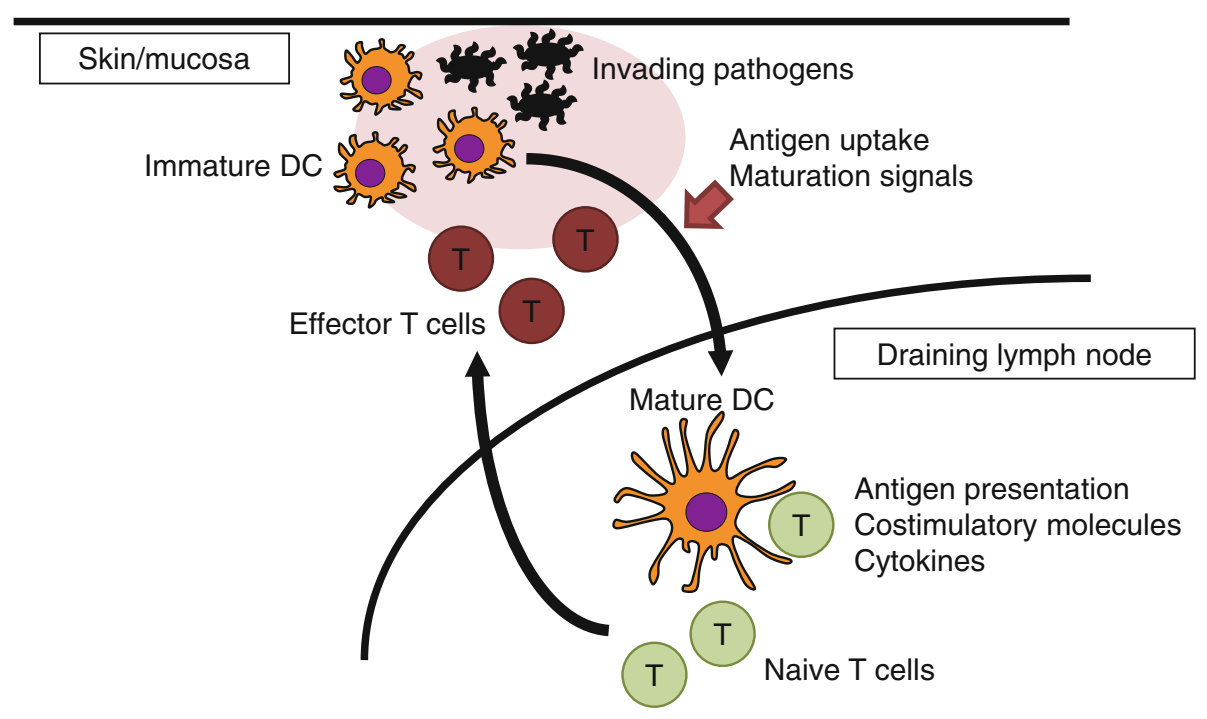

Fig. 1 Dendritic cells (DCs) act as an essential link between the innate and the adaptive immune systems. DCs reside in peripheral tissues in an immature form. Upon invasion of a pathogen, DCs capture antigens and receive maturation signals from these pathogens. DCs then migrate to the draining lymph node, where they become a

invade the tissue, DCs capture antigens and receive maturation signals from these pathogens through receptors of the innate immune system, such as Toll-like receptors (TLRs). DCs then migrate to the draining lymph node, where they become a mature form and initiate an adaptive immune response by presenting processed antigens on the HLA molecules to naive $T$ cells along with co-stimulatory molecules and cytokines that are required to induce effector and memory T cells [2]. Among APCs, DCs have the most potent capacity to activate antigen-specific $\mathrm{T}$ cells in vivo. Thus, DCs act as key controllers of antigen-specific $\mathrm{T}$ cell responses. Ralph Steinman, who discovered DCs in 1973 [3], was awarded the Nobel Prize in Medicine or Physiology in 2011.

Dendritic cell-based tumor immunotherapy, which is also called "DC vaccine", aims to harness the power of DCs to induce tumor-specific effector T cells [4]. Molecular identification of tumor-specific antigens recognized by $\mathrm{T}$ lymphocytes and technical advances in ex vivo generation of human DCs enabled us to develop this treatment strategy. DC vaccines have shown higher clinical efficacy than other active immunization approaches in collective data of melanoma vaccine trials [5]. DC vaccines are generated from ex vivo-cultured DCs that are loaded with tumor antigens and stimulated with maturation signals (Fig. 2). In this process, many variables exist regarding the source of DCs, antigen preparations, and maturation signals (reviewed in [6]). The most commonly used source of DCs is monocyte-derived DCs (MoDCs), which are generated by culturing monocyte in the presence of granulocyte mature form and present processed antigens on the HLA molecules to naive $\mathrm{T}$ cells with co-stimulatory molecules and cytokines, resulting in differentiation of antigen-specific naive $\mathrm{T}$ cells into effector $\mathrm{T}$ cells. Effector T cells in turn migrate to the peripheral tissue, and attack the pathogen

macrophage colony-stimulating factor (GM-CSF) and interleukin 4. The route of administration is also important, since it determines the homing property of $\mathrm{T}$ cells activated by DC vaccines. To maximize clinical efficacy of DC vaccines, these variables should be optimized.

A large number of clinical trials of DC vaccines have been conducted for a variety of tumors including hematological malignancies. Overall, these trials have demonstrated that DC vaccines have excellent safety profiles, moderate immunological activity and mild clinical efficacy. Notably, a DC-based immunotherapy for advancedstage prostate cancer called Provenge (also known as sipuleucel-T), using enriched blood DCs cultured with a fusion protein of prostatic acid phosphatase and GM-CSF, extended overall survival by approximately 4 months in phase III trials [7], and was approved by the US Food and Drug Administration in 2010.

\section{Current status of DC-based immunotherapy for hematological malignancies}

Recent reports on DC-based immunotherapy for hematological malignancies are summarized in Table 1.

Acute myeloid leukemia

In the first report of a DC vaccine trial for AML, Fujii et al. [8] used HLA matched donor $\mathrm{CD} 34^{+}$progenitor cell-derived DCs pulsed with autologous leukemia cells in combination 
Fig. 2 A scheme of DC-based tumor immunotherapy. DC vaccines are generated from ex vivo-cultured DCs, which are most commonly differentiated from patient-derived monocytes by culture in the presence of granulocyte macrophage colony-stimulating factor (GMCSF) and interleukin 4. They are then loaded with tumor antigen, stimulated with maturation signals and administered to the patient. There are many variables regarding the source of DCs, tumor antigen preparations, maturation signals, and the route of administration

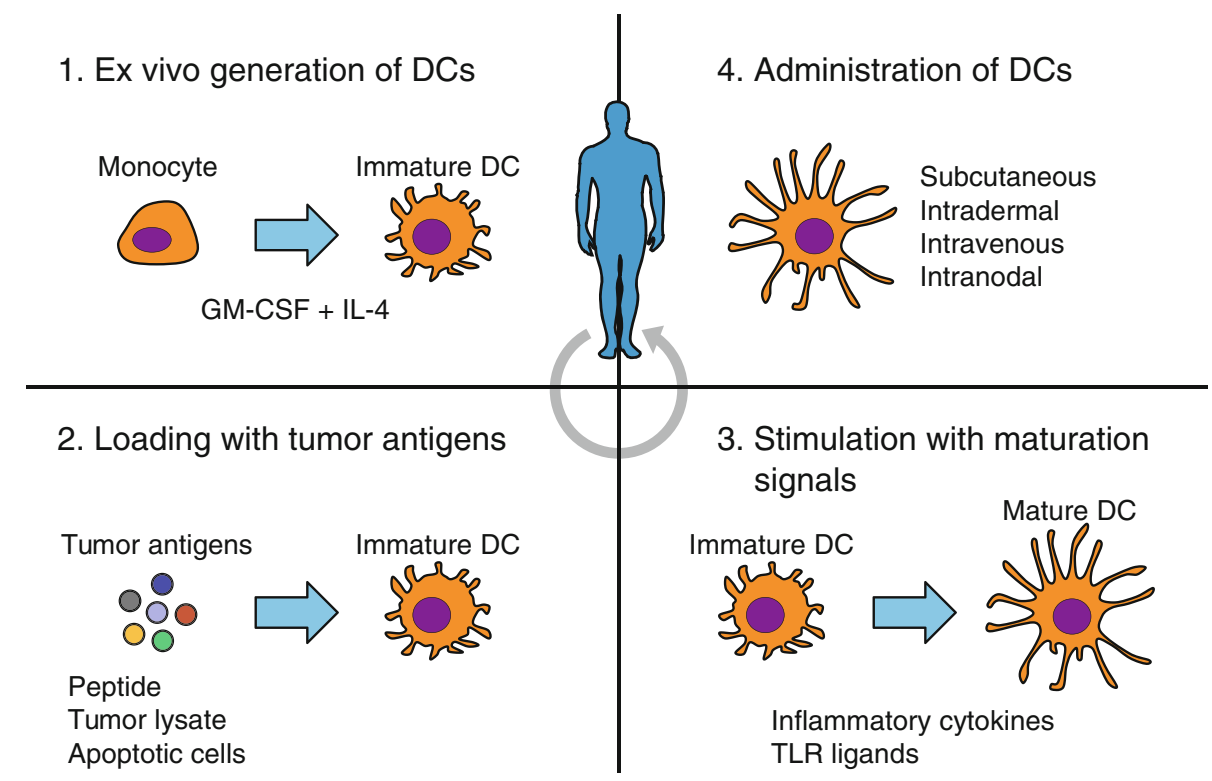

Myeloid leukemia is unique in that leukemia blasts can be cultured in the presence of cytokines and differentiated to express DC-like properties for use in vaccine therapy. These cells are called "leukemic DCs". Clinical trials using leukemic DCs have also been reported [9, 10]. However, the generation of DCs was feasible only in a limited number of patients, and even in patients with successful generation of DCs, the vaccine could not induce clinically relevant immune responses [9]. This may be due to poor immunostimulatory activity of leukemic DCs compared with MoDCs [11], providing a rationale for use of MoDCs in immunotherapy of AML.

Lee et al. [12] reported the first study of MoDC-based immunotherapy for AML relapsed after autologous peripheral blood SCT. The patients had high tumor burden, and the disease progressed rapidly after vaccination, although immune responses were induced. In contrast, a DC vaccine for AML in the minimal residual disease setting was reported by Van Tendeloo et al. [13], including 8 patients in complete remission with elevated WT1 mRNA levels and 2 in partial remission (PR), treated with fulllength WT1 mRNA-electroporated DCs. Molecular remission was achieved in 5 including the 2 PR patients after vaccination. Clinical responses were correlated with increases in WT1-specific $\mathrm{CD} 8^{+} \mathrm{T}$ cells. This study indicates that vaccine as a postremission treatment may prevent full relapse in patients with minimal residual disease.

We recently reported two clinical studies of MoDCbased immunotherapy for elderly patients with AML in morphologic remission [14, 15]. In one study, we administered MoDCs that engulfed autologous apoptotic leukemia cells and activated by OK-432 [14]. Of the 4 treated patients, we observed DC vaccine-induced immune responses in 2 patients with prolonged periods of disease with primed $\mathrm{T}$ cells for patients relapsed after allogeneic stem cell transplantation (SCT). A transient but objective clinical response was observed after injection of DCs and $\mathrm{T}$ cells. 
stabilization. In an HLA-A*24:02-positive patient, induction of $\mathrm{CD}^{+} \mathrm{T}$ cell responses to HLA-A*24:02-restricted WT1- and hTERT-peptides was observed, indicating crosspresentation of endocytosed leukemia cell antigens by DCs. In another study, we administered MoDCs pulsed with zoledronate and an HLA-A*24:02-restricted modified WT1 peptide, which has higher affinity to the HLA molecule than the natural WT1 peptide [15]. Of the 3 treated patients, 2 patients showed DC vaccine-induced anti-WT1 $\mathrm{CD}^{+} \mathrm{T}$ cell responses with transient periods of disease stabilization. Notably, $\mathrm{CD}^{+} \mathrm{T}$ cells reactive to the WT1 natural peptide persisted longer than those reactive to the modified WT1 peptide after the termination of DC vaccinations, implying that the former survived owing to antigenic stimulation by the WT1 natural peptide derived from endogenous residual leukemia cells.

Collectively, these studies indicate that MoDC-based immunotherapy is immunogenic even in elderly patients with AML after remission-induction therapy and warrant further study of this treatment strategy.

\section{Malignant lymphoma}

In lymphoma, DC vaccines have been tested particularly in indolent non-Hodgkin lymphoma, because the features of the disease, such as slow growth and incurability by chemotherapy [16], seem suitable to an active immunotherapeutic strategy.

In the first report of a clinical study of DC-based immunotherapy for lymphoma, four patients with follicular B cell lymphoma were treated with DCs enriched from leukapheresis product and pulsed ex vivo with tumor-specific idiotype protein [17]. In this study, all patients developed antitumor cellular immune responses with three patients showing clinical responses, such as complete or partial tumor regression and the disappearance of molecularly detectable disease after the vaccination.

Di Nicola et al. [18] reported a clinical study of DC vaccine for indolent B cell lymphoma using DCs loaded with killed autologous tumor cells that had been subjected to heat shock. Use of the whole autologous tumor cells as the source of antigen has the advantage of widening of the spectrum of target tumor-associated antigens including patient-specific mutated antigens, and stress stimuli such as heat shock have been shown to increase the immunogenicity of tumor cells [19]. Of the 18 patients with relapsed follicular or lymphoplasmacytic lymphoma, 6 patients had objective clinical responses including 3 continuous complete responses, and 8 patients had stable disease with a median follow-up of 50.5 months. Interestingly, the clinical responses were associated with a reduction in $\mathrm{CD} 4^{+}$ $\mathrm{CD}^{2} 5^{+}$Foxp3 $^{+}$regulatory $\mathrm{T}$ cells (Tregs), an increase in natural killer cells, and maturation of lymphocytes to the effector memory stage, suggesting that DC vaccinations have modulatory effects on multiple components of the immune system.

Multiple myeloma

Multiple myeloma also has similar clinical features to indolent lymphoma, such as slow growth and incurability by chemotherapy. Thus, myeloma is another attractive target of DC vaccines.

The most commonly used antigen to immunize myeloma patients is patient-specific idiotype protein. DC vaccines using idiotype protein as antigen have been reported [20-26]. Yi et al. [25] reported a clinical trial of intranodal injection of idiotype- and keyhole limpet hemocyaninpulsed, CD40 ligand-matured MoDCs into 9 patients with smoldering or stable myeloma. All patients mounted idiotype-specific interferon- $\gamma \mathrm{T}$ cell responses, including idiotype-specific cytotoxic $\mathrm{T}$ lymphocyte responses in 5 patients. Four patients had stable disease at 5-year followup.

A clinical trial using DC/tumor fusion cells was also reported in myeloma [27]. Fusion of DCs and tumor cells is one of the efficient methods to load DCs with the whole tumor antigens. Vaccination resulted in expansion of antimyeloma T cells in 11 of 15 evaluable patients, and disease stabilization in 11 of 16 evaluable patients with advanced disease.

Another remarkable approach to immunotherapy for myeloma is use of $\alpha$-galactosylceramide ( $\alpha$-GalCer)-pulsed DCs for vaccination to activate natural killer T (NKT) cells [28]. NKT cells recognize lipid antigens presented on the CD1d molecule, which is commonly expressed on myeloma cells. In a clinical trial, where $\alpha$-GalCer-pulsed MoDCs were administered in combination with low-dose lenalidomide to patients with asymptomatic myeloma, 3 of 4 patients with measurable disease had a reduction in $\mathbf{M}$ protein. This approach can be applied to other hematological malignancies, such as leukemia and lymphoma, as CD1d expression is observed on tumor cells in these diseases [29].

\section{Future perspectives in DC-based immunotherapy for hematological malignancies}

Eighteen years have passed since the first clinical trial of DC vaccine in lymphoma was reported in 1996. To date, DC-based tumor immunotherapy has been intensively investigated as an attractive vaccination approach, demonstrating a superior capacity to induce antitumor immune response in tumor patients, compared with other active immunization modalities [5]. However, the rate of 
objective tumor response remains low, even in patients with an induced antitumor response. One of the reasons for this poor clinical outcome is that most clinical trials of DC vaccine have been performed in end-stage patients with high tumor burden and/or a compromised immune system owing to intensive treatment history of chemotherapy. To establish a role for DC vaccines in the treatment of hematological malignancies, we need to define patient population who can obtain clinical benefit from DC vaccine. Lessons learned from clinical trials so far are that antitumor immune response is more likely to be induced in patients with less tumor burden, and that it takes several weeks or even months for DC vaccine-induced antitumor immune responses to manifest as a clinical response. Thus, it is conceivable that patients with indolent disease in remission can obtain most clinical benefit from DC vaccine. Remarkably, the excellent safety profile of DC vaccines is the advantage for use in this patient population. Clinical trials for this patient population should be further explored.

To enhance clinical efficacy of DC vaccines, combination therapy with other immunomodulatory agents is a promising approach. The presence of a DC vaccineinduced antitumor $\mathrm{T}$ cell response without an objective tumor response indicates that antitumor activity of the $\mathrm{T}$ cells is hindered by tumor-associated immunosuppressive mechanisms. Recent clinical trials revealed that immune checkpoint inhibitors, such as anti-CTLA-4 [30], anti-PD-1 [31], and anti-PD-L1 [32] monoclonal antibodies, alone or in combination [33] exhibit substantial tumor regressions even in patients with advanced-stage tumor. These therapies are good candidates for combination with DC vaccines, as these agents have the potential to rescue DC vaccine-induced antitumor $\mathrm{T}$ cells disabled by tumors. In this regard, an anti-CCR4 antibody for treatment of adult $\mathrm{T}$ cell leukemia (mogamulizumab) is another promising agent for use with DC vaccines, as it depletes Tregs in patients with cancer, especially CCR4-expressing effector Tregs that have the most potent suppressive activity [34].

In conclusion, DC vaccines will celebrate coming of age in a few years. Accumulating data in clinical trials have revealed advantages and disadvantages of this immunotherapeutic modality. Based on these data, how to make best use of DC vaccines to provide clinical benefit in patients with hematological malignancies should be further investigated in future clinical trials.

\section{References}

1. Banchereau J, Steinman RM. Dendritic cells and the control of immunity. Nature. 1998;392:245-52.
2. Ito T, Liu YJ, Kadowaki N. Functional diversity and plasticity of human dendritic cell subsets. Int J Hematol. 2005;81:188-96.

3. Steinman RM. Decisions about dendritic cells: past, present, and future. Annu Rev Immunol. 2012;30:1-22.

4. Palucka K, Banchereau J. Cancer immunotherapy via dendritic cells. Nat Rev Cancer. 2012;12:265-77.

5. Banchereau J, Palucka AK. Dendritic cells as therapeutic vaccines against cancer. Nat Rev Immunol. 2005;5:296-306.

6. Figdor CG, de Vries IJ, Lesterhuis WJ, Melief CJ. Dendritic cell immunotherapy: mapping the way. Nat Med. 2004;10:475-80.

7. Kantoff PW, Higano CS, Shore ND, Berger ER, Small EJ, Penson $\mathrm{DF}$, et al. Sipuleucel-T immunotherapy for castration-resistant prostate cancer. N Engl J Med. 2010;363:411-22.

8. Fujii S, Shimizu K, Fujimoto K, Kiyokawa T, Tsukamoto A, Sanada I, et al. Treatment of post-transplanted, relapsed patients with hematological malignancies by infusion of HLA-matched, allogeneic-dendritic cells (DCs) pulsed with irradiated tumor cells and primed T cells. Leuk Lymphoma. 2001;42:357-69.

9. Roddie H, Klammer M, Thomas C, Thomson R, Atkinson A, Sproul A, et al. Phase I/II study of vaccination with dendritic-like leukaemia cells for the immunotherapy of acute myeloid leukaemia. Br J Haematol. 2006;133:152-7.

10. Li L, Giannopoulos K, Reinhardt P, Tabarkiewicz J, Schmitt A, Greiner J, et al. Immunotherapy for patients with acute myeloid leukemia using autologous dendritic cells generated from leukemic blasts. Int J Oncol. 2006;28:855-61.

11. Draube A, Beyer M, Wolf J. Activation of autologous leukemiaspecific $\mathrm{T}$ cells in acute myeloid leukemia: monocyte-derived dendritic cells cocultured with leukemic blasts compared with leukemia-derived dendritic cells. Eur J Haematol. 2008;81: 281-8.

12. Lee JJ, Kook H, Park MS, Nam JH, Choi BH, Song WH, et al. Immunotherapy using autologous monocyte-derived dendritic cells pulsed with leukemic cell lysates for acute myeloid leukemia relapse after autologous peripheral blood stem cell transplantation. J Clin Apher. 2004;19:66-70.

13. Van Tendeloo VF, Van de Velde A, Van Driessche A, Cools N, Anguille S, Ladell K, et al. Induction of complete and molecular remissions in acute myeloid leukemia by Wilms' tumor 1 antigen-targeted dendritic cell vaccination. Proc Natl Acad Sci USA. 2010;107:13824-9.

14. Kitawaki T, Kadowaki N, Fukunaga K, Kasai Y, Maekawa T, Ohmori $\mathrm{K}$, et al. Cross-priming of $\mathrm{CD} 8(+) \mathrm{T}$ cells in vivo by dendritic cells pulsed with autologous apoptotic leukemic cells in immunotherapy for elderly patients with acute myeloid leukemia. Exp Hematol. 2011;39(424-433):e2.

15. Kitawaki T, Kadowaki N, Fukunaga K, Kasai Y, Maekawa T, Ohmori K, et al. A phase I/IIa clinical trial of immunotherapy for elderly patients with acute myeloid leukaemia using dendritic cells co-pulsed with WT1 peptide and zoledronate. Br J Haematol. 2011;153:796-9.

16. Salles G, Ghesquieres $H$. Current and future management of follicular lymphoma. Int J Hematol. 2012;96:544-51.

17. Hsu FJ, Benike C, Fagnoni F, Liles TM, Czerwinski D, Taidi B, et al. Vaccination of patients with B-cell lymphoma using autologous antigen-pulsed dendritic cells. Nat Med. 1996;2:52-8.

18. Di Nicola M, Zappasodi R, Carlo-Stella C, Mortarini R, Pupa SM, Magni M, et al. Vaccination with autologous tumor-loaded dendritic cells induces clinical and immunologic responses in indolent B-cell lymphoma patients with relapsed and measurable disease: a pilot study. Blood. 2009;113:18-27.

19. Feng H, Zeng Y, Whitesell L, Katsanis E. Stressed apoptotic tumor cells express heat shock proteins and elicit tumor-specific immunity. Blood. 2001;97:3505-12.

20. Reichardt VL, Okada CY, Liso A, Benike CJ, Stockerl-Goldstein $\mathrm{KE}$, Engleman EG, et al. Idiotype vaccination using dendritic 
cells after autologous peripheral blood stem cell transplantation for multiple myeloma: a feasibility study. Blood. 1999;93:2411-9.

21. Lim SH, Bailey-Wood R. Idiotypic protein-pulsed dendritic cell vaccination in multiple myeloma. Int J Cancer. 1999;83:215-22.

22. Titzer S, Christensen O, Manzke O, Tesch H, Wolf J, Emmerich $\mathrm{B}$, et al. Vaccination of multiple myeloma patients with idiotypepulsed dendritic cells: immunological and clinical aspects. Br J Haematol. 2000;108:805-16.

23. Reichardt VL, Milazzo C, Brugger W, Einsele H, Kanz L, Brossart P. Idiotype vaccination of multiple myeloma patients using monocyte-derived dendritic cells. Haematologica. 2003;88:1139-49.

24. Curti A, Tosi P, Comoli P, Terragna C, Ferri E, Cellini C, et al. Phase I/II clinical trial of sequential subcutaneous and intravenous delivery of dendritic cell vaccination for refractory multiple myeloma using patient-specific tumour idiotype protein or idiotype (VDJ)-derived class I-restricted peptides. $\mathrm{Br} \mathrm{J}$ Haematol. 2007;139:415-24.

25. Yi Q, Szmania S, Freeman J, Qian J, Rosen NA, Viswamitra S, et al. Optimizing dendritic cell-based immunotherapy in multiple myeloma: intranodal injections of idiotype-pulsed CD40 ligandmatured vaccines led to induction of type- 1 and cytotoxic T-cell immune responses in patients. Br J Haematol. 2010;150:554-64.

26. Rollig C, Schmidt C, Bornhauser M, Ehninger G, Schmitz M, Auffermann-Gretzinger S. Induction of cellular immune responses in patients with stage-I multiple myeloma after vaccination with autologous idiotype-pulsed dendritic cells. J Immunother. 2011;34:100-6.

27. Rosenblatt J, Vasir B, Uhl L, Blotta S, Macnamara C, Somaiya P, et al. Vaccination with dendritic cell/tumor fusion cells results in cellular and humoral antitumor immune responses in patients with multiple myeloma. Blood. 2011;117:393-402.

28. Richter J, Neparidze N, Zhang L, Nair S, Monesmith T, Sundaram $\mathrm{R}$, et al. Clinical regressions and broad immune activation following combination therapy targeting human NKT cells in myeloma. Blood. 2013;121:423-30.

29. Fujii S, Shimizu K, Hemmi H, Steinman RM. Innate Valpha14(+) natural killer T cells mature dendritic cells, leading to strong adaptive immunity. Immunol Rev. 2007;220:183-98.

30. Hodi FS, O'Day SJ, McDermott DF, Weber RW, Sosman JA, Haanen JB, et al. Improved survival with ipilimumab in patients with metastatic melanoma. N Engl J Med. 2010;363:711-23.

31. Ribas A. Tumor immunotherapy directed at PD-1. N Engl J Med. 2012;366:2517-9.

32. Brahmer JR, Tykodi SS, Chow LQ, Hwu WJ, Topalian SL, Hwu $\mathrm{P}$, et al. Safety and activity of anti-PD-L1 antibody in patients with advanced cancer. N Engl J Med. 2012;366:2455-65.

33. Wolchok JD, Kluger H, Callahan MK, Postow MA, Rizvi NA, Lesokhin AM, et al. Nivolumab plus ipilimumab in advanced melanoma. N Engl J Med. 2013;369:122-33.

34. Sugiyama D, Nishikawa H, Maeda Y, Nishioka M, Tanemura A, Katayama I, et al. Anti-CCR4 mAb selectively depletes effectortype FoxP3 + CD4 + regulatory $\mathrm{T}$ cells, evoking antitumor immune responses in humans. Proc Natl Acad Sci USA. 2013;110:17945-50.

35. Timmerman JM, Czerwinski DK, Davis TA, Hsu FJ, Benike C, Hao ZM, et al. Idiotype-pulsed dendritic cell vaccination for B-cell lymphoma: clinical and immune responses in 35 patients. Blood. 2002;99:1517-26 